\title{
Ensuring Quality in Biometric Systems
}

\author{
Md. Mahbubur Rahman ${ }^{1}$, Amit Karmaker ${ }^{1}$, Md.Mahmudul Hasan ${ }^{1}$ and Samsuddin \\ Ahmed $^{2}$ \\ ${ }^{1}$ Department of Computer Science and Engineering, \\ Patuakhali Science and Technology University, \\ Dumki-8602, Bangladesh. \\ ${ }^{2}$ Department of Computer Science and Engineering, \\ University of Chittagong (CU), Bangladesh. \\ ${ }^{1}$ mahbub.pstu@gmail.com, ${ }^{1}$ amitkarmaker06@gmail.com, ${ }^{1}$ zikucse@gmail.com \\ and ${ }^{2}$ sambd86@gmail.com
}

\begin{abstract}
Biometric system is using for personal recognition of people in many social and economical activities now a days. A good biometric trait should be measurable, distinctive and stable over time. Real-world deployment of biometric systems often has to contend with degraded signal quality and erratic behavior of the biometric data. For last few years biometric data quality measure become an important concern after poor pathological sample and other many causes. The user, sensor and environmental facts are causes to quality degradation of biometric system. This study approaches that have been used to extract additional information about the biometric data that can then be used to improve performance in degraded conditions and also discuss about the sensor and environmental facts. This study will also discuss how this problem can be overcome to maintain the quality in biometric system with a special emphasis on face, fingerprint, iris modalities with different organizational standards.
\end{abstract}

Keywords: Biometrics, security, quality assessment, sample quality.

\section{Introduction}

Biometric system is the process of authentication or identification based on persons anatomical or behavioral unique characteristics. It refers to automatic recognition of an individual based on anatomical (e.g., fingerprint, face, iris, hand geometry, ear, palm print) or behavioral characteristics (e.g., signature, gait, keystroke dynamics). Biometrics offers greater convenience and several advantages over traditional security methods based on something that you know (e.g. password, PIN) or something that you have (e.g. card, key, etc.). In biometric systems, users don't have to remember passwords or PINs, which can be forgotten, or carry cards or keys, which can be stolen. Nowadays, biometric recognition is used in many governments and civilian applications such as e-Passports, ID cards, or border control. There are a number of factors that can affect the quality of biometric signals, and there are numerous roles of a quality measure in the context of biometric.

\section{Biometric System Performance}

In Biometric system the performance of a system is highly depend on signal quality by which the biometric data is capture and the signal quality have many parameter which effects on signal. Biometric data can be capture by two ways one is controlled environment and other is uncontrolled environment. In controlled environment there is slightly degradation of quality for processing issue but most of quality degradation is in uncontrolled situation. 
In controlled situation there have strict description and design of system parameter by which no/slightly quality degradation. But for uncontrolled situation different new era can come to our systems data and that affect the system performance. The researchers have tested the system by intentionally corrupting the parameter and show the resulting quality degradation.

\section{Quality Issue to Biometric Data}

Biometric data quality is good if it is suitable for person's recognition. Quality based biometric systems have low false positive and low false negative error to identify persons. Different organization declares different standards to maintain biometric sample quality. According to ISO there are three major considerations they are character, fidelity and utility. The character of the sample source and the fidelity of the processed sample contribute to, or similarly detract from, the utility of the sample. For security, identification, and for other purpose it is very sensitive to use biometric data. If there have any quality degradation to sample then one person's data can be merging with others and can causes to great harm to some resource which is actually undesirable. It must be distinctive and quality based in every step.

\section{Factors Influencing Biometric Quality}

There have different factors that influence to the quality of biometric system. Eric Kukula and his colleagues' framework and other previous research show some factors. The study shows some important factors that category into four basic type they are user related, user-sensor interaction, acquisition sensor, and processing-system factors.

\section{User-Related Factors}

The user inherent behavior that effect to system performance. The user action is not same in every time but the system is work with a fixed mode of user. According to different phenomena the user related factor are basically three type are (1).Physical Consider the age, gender.(2)Behavioral-Tiredness, distraction, cooperatively, motivation, nervousness ,Distance, eyes closed, facial expression, pose, gaze Pressure against the sensor Inconsistent contact etc. The user related factors impact on character.

\section{User-Sensor Interaction Factors}

Users impact will depend on the level of control of the environment, the acquisition itself, and whether the acquisition physically takes place in controllable premises. In many applications, biometric data is acquired in less than ideal conditions, such as by surveillance cameras or portable hand-held devices. The user must interact with sensor perfectly if the user is not aware to interact with sensor then the system performance is degrade. The common user sensor interaction factors are (1).Environmental Indoor/outdoor operation, Background, object occlusion Temperature, humidity, Illumination, light, reflection, Ambient noise, distance. (2.) Operational - User familiarity, Feedback of acquired data, Supervision by an operator, Sensor cleaning, physical guides, Ergonomics, Time between acquisitions. These factors effect on fidelity.

\section{Acquisition Sensor Factors}

The sensor is actually used for interact with the user. But now a days there have many low costs sensor device are use for biometric data acquisition this is impact on fidality.The common sensor related factors are Ease of use and maintenance, Acquisition area, 
physical robustness, Resolution, noise, input/output, linearity,

dynamic range, Acquisition time etc.

\section{Processing-System Factors}

The system related factors are related how a biometric data is processed once it is accepted. The common system related factors are Exchange and storage format of data, Processing algorithms, Data compression, Network etc. This is impact on fidelity.

\section{Biometric Quality Assurance Process}

Biometric sample's quality maintenance is an important factor to ensure the success of the biometric system. Which are summarized in three points of action can be identified: i) the capture point ii) the quality assessment algorithm itself, and iii) the system that performs the recognition process.

\section{Capture Point}

In the capture point capture the biometric data according to specific standard. The common guide lines are supervision of an operator, adequate sensor with enough capability and new feature, real-time feedback with enhanced GUI, proper user interaction by physical positioning and user friendly process, adequate environment by light, background, and moisture control and finally suggested to use certified sensor.

\section{The Quality Assessment Algorithm}

Quality assessment algorithm is used to measuring different factors affecting the quality of biometric traits.Quality assessment algorithms have been developed mainly for fingerprint images and recently, for iris, voice, face and signature signals. The common guidelines are maintain time response vs. good quality tradeoff in real-time operation, problem corrective action in acquisition loop, and using certified quality assessment algorithm.

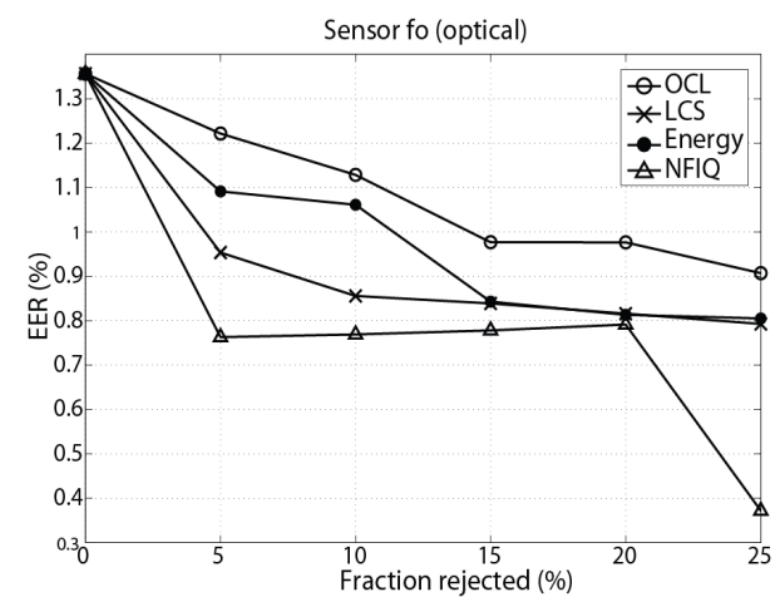

Figure 1. Evaluating the Utility of Four Fingerprint Quality Measures

System

The system related quality assurance is to maintain a quality based system in processing, storing, template management and quality based decision making. To maintain quality based processing by additional enhancement, alternative feature extraction and different matching algorithm. The system can use quality based fusion by combining different biometric traits and algorithm. The system can update the template 
after a successful pass of a user to overcome the system limitation in future as the biometric data may changing in some period of life of person if update the template in time to time the system can overcome fidelity problem in future. The certified system can be used to obtain a more strong system.

\section{Incorporating Quality Measure in Biometric System}

The incorporation of quality measures in biometric systems is an active field of research, with many solutions proposed. This study shows different uses of sample quality measures in the context of biometric systems. Summarizing different uses of sample quality measures in this context.

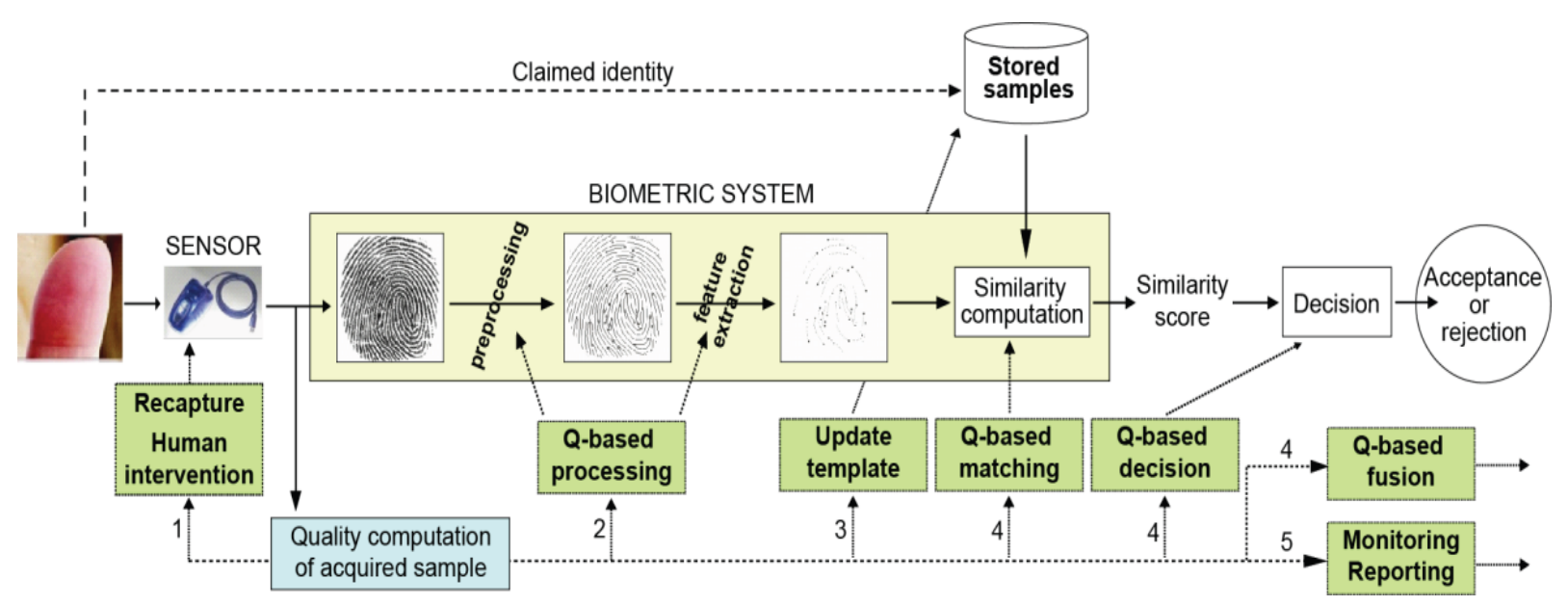

Figure 2. The Figure Shows the Quality Measures in Biometric System

The recapture loop implements an "up to three attempts" policy, giving feedback in each subsequent acquisition to improve quality.

Quality-specific enhancement algorithms; conditional execution of processing chains, including specialized processing for poor quality data, extraction of features robust to the degradation that the signal is suffering, extraction of features from useful regions only, ranking of extracted features based on the quality of local regions.

Storage of multiple samples representing the variability associated with the user, update of stored samples with ones of better quality captured during the operation of the system.

Use of different matching or fusion algorithms to adjustment of the sensitivity of the matcher or fusion algorithm, quantitative indication of the reliability of the acceptance or rejection decision, quality-driven selection of data sources to be used for matching or fusion, use of soft-biometric traits (age, height, sex, etc.) to assist in the recognition task.

Monitoring and reporting across the different parts of the system to identify problems that lead to poor quality signals and initiate corrective actions. Different aspects that can be monitored and reported include signal quality. This process can assess signal quality according to some factors, they are Application, Site or terminal, Capture device, Subject, Stored template, Biometric input etc. 


\section{Organizational Standardizing Biometric Quality}

It should be noted that adhesion to standards for sensors, software, interfaces, etc. is recommended throughout the quality assurance process. With the use of standards, great flexibility and modularity is obtained, as well as fast technology interchange, sensor and system interoperability and proper interaction with external security systems. As biometric technology is extensively deployed, a common situation is the exchange of information between several multi-vendor applications of different agencies, involving heterogeneous equipment, environments and/or locations.

Different standards organizations and other bodies are working in biometric standards development as example International Electro-technical Commission(IEC), International Organization for Standardization, Committee 1 on Information Technology, Subcommittee 37 for Biometrics(ISO-JTC1/SC37), American National Standards Institute( ANSI), International Civil Aviation Organization(ICAO), International Committee for Information Technology Standards Technical Committee M1 on Biometrics(INCITS M1), Biometric Consortium(BC), Biometric Center of Excellence(BCOE), Biometrics Identity Management Agency(BIMA), International Biometric Group(IBG), International Biometrics and Identification Association(IBIA).Some important and latest biometric standards are shown on below.

ANSI/NIST-ITL 1-2000: Supports the exchange of biometric data, including fingerprints, faces, scars, marks, and tattoos, between law enforcement and related criminal justice agencies.

ANSI/NIST-ITL 1-2007/2-2008: Defines a common format for exchanging and storing a variety of biometric data including faces, fingerprints, palm prints, irises, voices, and written signatures.

BioAPI (Biometric Application Programming Interface) defines the architecture and necessary interfaces to allow biometric applications to be integrated from different vendors.

FBI-EBTS (FBI Electronic Biometric Transmission Specification): Supports the exchange of biometric data with the US FBI.

ISO/IEC-19794: A common format to exchange and store a variety of biometric data.

ISO/IEC 29794-1/4/5: Enables harmonized interpretation of quality scores from different vendors, algorithms, and versions by setting key factors to define quality in different biometric traits.

Annex to ISO/IEC-19794-5: Includes recommendations for taking photographs of faces for e-passport and related applications and includes indications about lighting, camera arrangement, and head positioning.

\section{Conclusion}

Performance of biometric systems is dependent on the quality of the acquired input samples. If quality can be improved, either by sensor design, by user interface design, or by standards compliance, better performance can be realized. For those aspects of quality that cannot be designed-in, an ability to analyze the quality of a live sample is needed. This is useful primarily in initiating the reacquisition from a user, but also for the realtime selection of the best sample, and the selective invocation of different processing 
methods. It is the key component in quality assurance management, and because quality algorithms often embed the same image (or signal) analyses needed to assess conformance to underlying data interchange standards, they can be used in automated image screening applications. Quality analysis is a technical challenge because it is most helpful when the measures reflect the performance sensitivities of one or more target biometric matchers. This study has presented a framework to meet the challenge in biometric system also discuss the constraint of biometric system that affects the quality of Biometric systems quality. This study also discuss about different Standard organizational approach to maintain the quality of Biometric system.

\title{
References
}

[1] P. Grother and E. Tabassi, "Performance of Biometric Quality Measures", IEEE Trans. Pattern Analysis and Machine Intelligence, vol. 29, no. 4, (2007), pp. 531-543.

[2] A. K. Jain, B. Klare and U. Park, "Face Recognition: Some Challenges in Forensics", Proc. Int'l Conf. Automatic Face and Gesture Recognition (FG), IEEE, (2011).

[3] A. K. Jain and A. Kumar, "Biometrics of Next Generation: An Overview", Second Generation Biometrics, Springer, (2010).

[4] A. K. Jain, B. Klare and U. Park, "Face Recognition: Some Challenges in Forensics", Proc. Int'l Conf. Automatic Face and Gesture Recognition (FG), IEEE, (2011).

[5] R. Youmaran and A. Adler, "Measuring Biometric Sample Quality in Terms of Biometric Information", Proc. Biometric Consortium Conf.: Special Session on Research at the Biometrics Symp., IEEE, (2006).

[6] E. P. Kukula, M. J. Sutton and S. J. Elliott, "The Human- Biometric-Sensor Interaction Evaluation Method: Biometric Performance and Usability Measurements", IEEE Trans. Instrumentation and Measurement, vol. 59, no. 4, (2010), pp. 784-791.

[7] N. D. Kalka, "Estimating and Fusing Quality Factors for Iris Biometric Images", IEEE Trans. Systems, Man and Cybernetics, Part A: Systems and Humans, vol. 40, no. 3, (2010), pp. 509-524.

[8] F. Alonso-This study, "A Comparative Study of Fingerprint Image Quality Estimation Methods", IEEE Trans. Information Forensics and Security, vol. 2, no. 4, (2007), pp. 734-743.

[9] M. Theofanos], "Biometrics Systematic Uncertainty and the User", Proc. IEEE Conf. Biometrics: Theory, Applications and Systems (BTAS), IEEE, (2007), pp. 1-6.

[10] K. E. Wertheim, "Human Factors in Large-Scale Biometric Systems: A Study of the Human Factors Related to Errors in Semiautomatic Fingerprint Biometrics", IEEE Systems J., vol. 4, no. 2, (2010), pp. 138-146.

[11] 11.D. D. Amato, N. Hall and D. McGarry, "The specification and measurement of face image quality", Performance Testing Conference, IBPC, http://www.nist.gov/itl/iad/ig/ibpc2010.cfm, (2010).

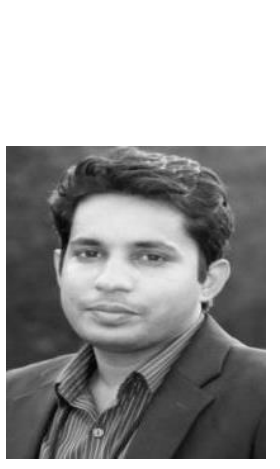

\begin{abstract}
Authors
Md.Mahbubur Rahman, received his B.Sc.Engineering degree in computer science and engineering from Patuakhali Science and Technology University in 2011 and working as a Lecturer in computer science and engineering at one of the top most private Universities in Bangladesh named Bangladesh University of Business and Technology (BUBT). His research interests are Data mining, Search Engine optimization, Biometric system, Network Security.
\end{abstract}

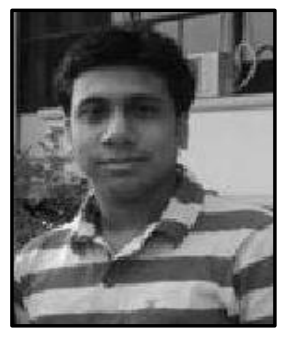

Amit Karmaker, received his B.Sc. Engineering degree in computer science and engineering from Patuakhali Science and Technology University in 2011 and working as a Assistant Programmer at a State Owned Top ranked Bank named Janata Bank, Bangladesh. His research interests are Wireless Networking, Near Field Communication, Data mining, Search Engine optimization, Biometric system, Network Security. 


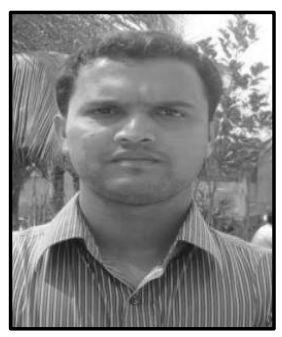

Md Mahmudul Hasan, has completed his B.Sc Engg. in Computer Science and Engineering from Patuakhali Science and Technology University in 2011 and working as an Instructor in Computer Studies an Satkhira Primary Teachers Training Institute, Bangladesh. His research interest is Semantic web, Routing algorithm, High dimensional data mining.

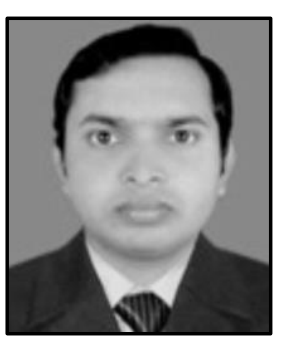

Samsuddin Ahmed has been lecturing in CSE since mid of 2010. $\mathrm{He}$ is in Computer Science and Engineering from University of Chittagong with highest CGPA till date. His under-grade Thesis was on "Handling Uncertainties in Spatial Feature Extraction". His hobbies include thinking about underlying mathematical formulations in natural phenomena. His research interests include data and image mining, Semantic Web, Business Intelligence, Spatial Feature Extraction etc. He is now serving one of the top most private Universities in Bangladesh named Bangladesh University of Business and Technology (BUBT). 
International Journal of Security and Its Applications

Vol. 9, No. 4 (2015) 